\title{
ASYMPTOTIC PROPERTIES OF ROOTED 3-CONNECTED MAPS ON SURFACES
}

\author{
EDWARD A. BENDER, ZHICHENG GAO, L. BRUCE RICHMOND \\ and NICHOLAS C. WORMALD
}

(Received 5 March 1993)

Communicated by L. Caccetta

\begin{abstract}
In this paper we obtain asymptotics for the number of rooted 3-connected maps on an arbitrary surface and use them to prove that almost all rooted 3-connected maps on any fixed surface have large edge-width and large face-width. It then follows from the result of Roberston and Vitray [10] that almost all rooted 3-connected maps on any fixed surface are minimum genus embeddings and their underlying graphs are uniquely embeddable on the surface.
\end{abstract}

1991 Mathematics subject classification (Amer. Math. Soc.): 05C10, 05C30, $05 \mathrm{C} 15$.

\section{Introduction}

We begin with some definitions:

- A map is a connected graph $G$ embedded in a surface $S$ (a closed 2-manifold) such that all components of $S-G$ are simply connected regions, which are called faces. $G$ is called the underlying graph of $M$, and is denoted by $G(M)$. Loops and multiple edges are allowed in $G$.

- A map is rooted if an edge is distinguished together with a vertex on the edge and a side of the edge. All maps shall be rooted.

- We use Tutte's definition [11] of connectivity: a graph (or the corresponding map) is $k$-connected (abbreviated $\mathrm{k}-\mathrm{c}$ ) if the girth is at least $k$ and it requires removing at least $k$ vertices to separate the graph.

Research of second and third authors supported by the NSERC under Grant A-4067.

Research of fourth author supported by the Australian Research Council.

(C) 1996 Australian Mathematical Society 0263-6115/95 $\$ A 2.00+0.00$ 
- By a cycle in a map, we mean a simple closed curve consisting of edges of the map. A cycle is called separating if deleting it separates the underlying graph and is called facial if it bounds a face of the map.

- A cycle is called contractible if it is homotopic to a point; otherwise it is called non-contractible and denoted by $n c$-cycle.

- The edge-width of a map $M$, denoted by ew $(M)$, is the length of a shortest nc-cycle of $M$. The face-width (also called representativity in [10]) of a map $M$, denoted by fw $(M)$, is the minimum of $|G(M) \cap C|$ taken over all non-contractible simple closed curves $C$ that lie in the surface and contain no vertices of $G(M)$. It is easily seen that $\operatorname{ew}(M) \geq \mathrm{fw}(M)$ for any map $M$.

We will prove the following theorem and its corollaries.

THEOREM 1. Let $t_{g}$ and $p_{g}$ be the constants defined in Theorem 1 of [1]. The number of 3-connected maps on a surface of Euler characteristic $\chi=2-2 g$ is given asymptotically by

$$
\begin{array}{ll}
t_{g}(9 n)^{5 \times / 4} 4^{n} & \text { on orientable surfaces, and } \\
p_{g}(9 n)^{5 \times / 4} 4^{n} & \text { on non-orientable surfaces. }
\end{array}
$$

In the following corollaries, 'almost all' means that the fraction of maps having the property approaches 1 as $n \rightarrow \infty$.

COROLlARY 1. Almost all n-edged 3-connected maps on a given surface have face-width greater than $\delta \log n$ for some constant $\delta>0$.

COROLLARY 2. Almost all n-edged 3-connected maps on a given surface are not hamiltonian.

While interesting in itself, the theorem is also important because it shows that the number of 3-c maps on a surface 'grows normally.' This concept is defined as follows:

- Let $\mathscr{F}$ be some family of maps and let $\mathscr{F}_{n}(S)$ be the set of $n$-edged maps in $\mathscr{F}$ that lie on a surface $S$. We say that $\mathscr{F}$ grows normally if

$$
\left|\mathscr{F}_{n}(S)\right| \sim A(S, \mathscr{F}) n^{-5 \chi / 4} \rho(\mathscr{F})^{n}
$$

for some $A(S, \mathscr{F})$ and $\rho(\mathscr{F})$, where $\chi$ is the Euler characteristic of $S$ and the limit is taken through those $n$ for which $\mathscr{F}_{n}(S) \neq \emptyset$.

A variety of families of maps exhibiting normal growth are listed in [7]. Properties of such families are discussed in $[3,4]$ and imply the corollaries. 
The motivation for the first corollary may not immediately be apparent. Robertson and Vitray [10] have studied graph embeddings with large face-width. They have shown that they share many properties with planar embeddings. For example, if $M$ is a map of genus $g$ which has face-width exceeding $2 g+2$, then it is the minimum genus embedding of $G(M)$, and if $G(M)$ is also 2-connected, then any other embedding of $G(M)$ of genus $g$ is obtained from $M$ by a sequence of '2-switchings' (defined by Whitney [13] who proved the planar case). It follows from this that, if a 3-connected map of genus $g$ has face-width exceeding $2 g+2$, then $G(M)$ has a unique embedding of genus $g$.

After establishing the connection between certain types of quadrangulations and 3-connected maps, we will focus on quadrangulations for two sections. In the last section, we use these results to prove Theorem 1 and its corollaries. To avoid too many technical details, we shall only prove our results for maps on orientable surfaces. Similar arguments work for maps on non-orientable surfaces.

\section{Quadrangulations and 3-connected maps}

- A bipartite quadrangulation is a map whose underlying graph is bipartite and whose faces are all quadrangles. All quadrangulations shall be bipartite.

- A quadrangulation is called near-simple if it has no contractible 2-cycles and no contractible non-facial 4-cycles, and is called simple if it has no 2-cycles and all 4-cycles are facial.

The following lemma connects these concepts with 3-connected maps.

LEMMA 1. There is a bijection $\phi$ between $n$-edged maps and $n$-faced quadrangulations, such that $\mathrm{fw}(M)=\operatorname{ew}(\phi(M)) / 2$. Furthermore, $\phi(M)$ simple implies $M$ 3-connected, which implies that $\phi(M)$ is near-simple.

PROOF. The proof of the first statement is a straightforward extension of the bijection on the sphere given by Brown [6]: For any map $M$, place a vertex in each face and join it to the vertices on the boundary of the face through every corner and remove all the original edges of $M$. This gives a bipartite quadrangulation $Q$, whose root corner can be chosen the same as the root corner of $M$. This is clearly a bijection, and any nc-cycle of length $2 k$ in $Q$ intersects $G(M)$ in exactly $k$ vertices.

Suppose now that $\phi(M)$ is not near-simple, that is, $\phi(M)$ has either a contractible 2-cycle or a contractible non-facial 4-cycle. Then in the former case, one vertex in the 2-cycle is a cut vertex of $M$, and in the latter case, two non-adjacent vertices in the 4-cycle form a cut-pair of $M$. So $M$ 3-connected implies $\phi(M)$ near-simple.

Now suppose that $M$ is not 3-connected; then the edges of $M$ can be partitioned into two classes, say blue and red, such that only two vertices, say $v_{1}$ and $v_{2}$, are 
incident to both blue and red edges. Pick a face $f_{1}$ incident to both blue and red edges at $v_{1}$. Tracing around $f_{1}$ from the blue edge, we must eventually reach a red edge. This must occur at $v_{1}$ or $v_{2}$. If it is at $v_{1}$, then $v_{1}$ and $f_{1}$ have multiple incidence, and this gives a 2-cycle in $\phi(M)$. If it is at $v_{2}$, we can then pick another such face $f_{2}$ and repeat the process, thereby finding two faces $f_{1}$ and $f_{2}$ both incident to $v_{1}$ and $v_{2}$. They form a non-facial 4-cycle in $\phi(M)$. So $\phi(M)$ being simple implies that $M$ is 3-connected.

Our approach is similar to that used in [5]. We obtain asymptotics for the number of near-simple quadrangulations and then show that almost all near-simple quadrangulations are simple. It then follows from Lemma 1 that the numbers of near-simple quadrangulations, simple quadrangulations and 3-connected maps are all asymptotically the same.

\section{Enumerating near-simple quadrangulations}

On the orientable surface of genus $g=1-\chi / 2$, with $x$ marking the number of faces, define the following generating functions:

$Q_{g}(x)$ : quadrangulations,

$\hat{Q}_{g}(x)$ : quadrangulations without contractible 2-cycles,

$Q_{g}^{*}(x)$ : near-simple quadrangulations.

Let $R_{1}=\sqrt{1-12 x}, R_{2}=\sqrt{1-27 x / 4}$, and $R_{3}=\sqrt{1-4 x}$.

It follows from Lemma 1 above, [2] and [1, Theorem 1 and Lemma 3] that $Q_{g}(x)$ is algebraic and has a Laurent series expansion in $R_{1}$ :

$$
Q_{g}(x)= \begin{cases}\frac{1}{3}-\frac{4}{3} R_{1}^{2}+(8 / 3) R_{1}^{3}\left(1+O\left(R_{1}\right)\right), & \text { if } g=0, \\ A_{g} R_{1}^{3-5 g}\left(1+O\left(R_{1}\right)\right), & \text { if } g>0\end{cases}
$$

where the $A_{g}$ 's are constants. We will prove similar results for $\hat{Q}_{g}$ and $Q_{g}^{*}$ :

THEOREM 2. $\hat{Q}_{g}(x)$ and $Q_{g}^{*}(x)$ are algebraic and have the following Laurent series expansions:

$$
\begin{gathered}
\hat{Q}_{g}(x)= \begin{cases}\frac{1}{3}-\frac{4}{9} R_{2}^{2}+\frac{8}{27 \sqrt{3}} R_{2}^{3}\left(1+O\left(R_{2}\right)\right), & \text { if } g=0, \\
\left(A_{g} / 3\right)\left(R_{2} / \sqrt{3}\right)^{3-5 g}\left(1+O\left(R_{2}\right)\right), & \text { if } g>0,\end{cases} \\
Q_{g}^{*}(x)= \begin{cases}\frac{407}{4320}-\frac{14539}{64800} R_{3}^{2}+\frac{8}{729} R_{3}^{3}\left(1+O\left(R_{3}\right)\right), & \text { if } g=0, \\
\left(A_{g} / 9\right)\left(R_{3} / 3\right)^{3-5 g}\left(1+O\left(R_{3}\right)\right), & \text { if } g>0,\end{cases}
\end{gathered}
$$


where $A_{g}$ is given by (3.1). Moreover, the only possible finite singularities of $\hat{Q}_{g}(x)$ are at $x=4 / 27,-16 / 27$ and -4 and the only singularity of $Q_{g}^{*}(x)$ on its circle of convergence is at $x=1 / 4$.

ProOF. Lemma 1 allows us to convert statements about maps to statements about quadrangulations. Tutte's formula for $Q_{0}[12,(5.2)]$, can be written as

$$
1+Q_{0}(x)=\frac{4\left(1+2 R_{1}\right)}{3\left(1+R_{1}\right)^{2}} .
$$

Tutte also proved [12, p.257]

$$
\hat{Q}_{0}(x)=t(2-3 t), \quad \text { where } \quad x=t(1-t)^{2} \text { and } t(0)=0,
$$

from which the $g=0$ case of (3.2) follows. The formula for $Q_{0}^{*}(x)$ can be obtained from $Q_{N}^{*}(x, x)$ given by $[8,(6.24-25)]$. Thus, we need only consider the cases $g>0$.

We require some definitions.

- A contractible cycle in a non-planar map separates the map into a planar piece and a non-planar piece. The planar piece is called the interior of the cycle and we also say that the cycle contains anything in its interior. Since we usually draw a planar map such that the root face is the unbounded face, we define the interior of a cycle in a planar map to be the piece which does not contain the root face.

- A 2-cycle or 4-cycle is called maximal (minimal) if it is contractible and its interior is maximal (minimal).

It is important to note that, in any quadrangulation, all maximal 2-cycles have disjoint interiors, and that, in any non-planar quadrangulation without contractible 2-cycles, all maximal 4-cycles have disjoint interiors. (This is simpler than the planar case [8, p. 260].) Therefore, we can close all maximal 2-cycles in quadrangulations to obtain quadrangulations without contractible 2-cycles, and remove the interior of each maximal contractible 4-cycle to obtain near-simple quadrangulations. The process can be reversed and used to construct quadrangulations from near-simple quadrangulations.

To study $\hat{Q}_{g}$, we use an approach similar to that in [5]. All quadrangulations of genus $g>0$ can be divided into two classes according to whether or not the root face lies in the interior of some contractible 2-cycle.

For any quadrangulation in the first class, let $C$ be the minimal 2-cycle containing the root face. Cutting along $C$, filling holes with disks and closing those two digons, we obtain a general quadrangulation of genus $g$ and a planar quadrangulation with a distinguished edge. Taking the latter quadrangulation, cutting along all of its maximal 2-cycles and closing as before gives a quadrangulation without contractible 2-cycles, 
together with a set of planar quadrangulations extracted from within the maximal 2cycles. Thus the generating function for the first class is $2 u \hat{Q}_{0}^{\prime}(u) Q_{g}(x) /\left(1+Q_{0}(x)\right)$, where

$$
u=x\left(1+Q_{0}(x)\right)^{2} .
$$

For any quadrangulation in the second class, closing all maximal contractible 2cycles gives quadrangulations without contractible 2-cycles. Thus the generating function for this class is $\hat{Q}_{g}(u)$. Combining the two classes, we have $Q_{g}(x)=$ $\hat{Q}_{g}(u)+2 u \hat{Q}_{0}^{\prime}(u) Q_{g}(x) /\left(1+Q_{0}(x)\right)$ and so

$$
\hat{Q}_{g}(u)=\left(1-\frac{2 u \hat{Q}_{0}^{\prime}(u)}{1+Q_{0}(x)}\right) Q_{g}(x),
$$

which is the same as the first line of $[5,(4.1)]$. Using (3.1), (3.2) with $g=0$, and (3.7), we obtain (3.2) for $g>0$.

We now use a similar argument to derive $Q_{g}^{*}(x)$ from $\hat{Q}_{g}(x)$. For any quadrangulation without contractible 2-cycles, let $C$ be the maximal contractible 4-cycle containing the root face. Cutting along $C$ and filling holes with disks, we obtain

(a) a planar quadrangulation which has no 2-cycles and has a distinguished face other than the root face, and

(b) a quadrangulation of genus $g$ which, after the removal of the interiors of all maximal 4-cycles, gives a near-simple quadrangulation.

Note that

$$
v=v(x)=\frac{\hat{Q}_{0}(x)-2 x}{x}
$$

enumerates by the number of interior faces the planar quadrangulations having at least one interior face and having no 2-cycles. It follows from the construction that

$$
x^{2} \hat{Q}_{g}(x)=\left(x^{2} v^{\prime}(x)\right) \times\left((x / v) Q_{g}^{*}(v)\right)
$$

which gives

$$
Q_{g}^{*}(v)=\frac{v}{x v^{\prime}} \hat{Q}_{g}(x)
$$

Using (3.2), (3.8) and (3.9), we obtain (3.3) for $g>0$.

The sources of the singularities of $\hat{Q}_{g}(u)$ fall into three classes:

(1) singularities of $Q_{g}(x)$ that are carried over to $\hat{Q}_{g}(u)$ by (3.7),

(2) singularities that arise when (3.6) is solved for $x(u)$ to use in (3.7), and

(3) singularities of $\hat{Q}_{0}(u)$.

By [1, Lemma 3], the only possible singularities of $Q_{g}(x)$ are at $1 / 12$ and $-1 / 4$, the latter requiring $R_{1}(-1 / 4)=-2$. These lead to $u=4 / 27$ and $u=-4$, respectively. 
We now turn to the second source of singularities. Following [5], we rewrite (3.4) as

$$
27 u\left(1+R_{1}\right)^{3}+4\left(R_{1}-1\right)\left(2 R_{1}+1\right)^{2}=0
$$

and observe that this can be further rewritten as a polynomial equation in $x$ and $u$. After some algebra, one finds that the leading coefficient in $x$ vanishes at $u=-16 / 27$, and multiple roots can occur when $R_{1}=0$. This determines all possible singularities for $\hat{Q}_{g}$ in the second class. By (3.5), the last class corresponds to multiple roots $t(u)$ of $t(1-t)^{2}=u$, which leads to $u=4 / 27$.

The same sort of argument is used with (3.9) to study the singularities of $Q_{g}^{*}(v)$. Since $Q_{0}^{*}$ does not appear in that formula, the third source of singularities does not arise. The three possible singularities $x=4 / 27,-16 / 27$ and -4 of $\hat{Q}_{g}$ give only $v(x)=1 / 4,-3 / 8$ and $-3 / 4$ as possible singularities of magnitude less than 1 . We now turn to the second source of singularities. From (3.8) and (3.5) we have $v=t(1-2 t) /(1-t)^{2}$, where $t(0)=0$. A little algebra leads to

$$
t=\frac{2 v+1 \pm \sqrt{1-4 v}}{v+2}
$$

which has singularities at $v=1 / 4$ and $v+2=0$.

\section{Almost all near-simple quadrangulations are simple}

In this section, we prove

THEOREM 3. Almost all near-simple quadrangulations on any fixed surface are simple; that is, the ratio of the number of $n$-faced near-simple quadrangulations to $n$-faced simple quadrangulations approaches 1 as $n \rightarrow \infty$.

PROOF. We must prove that almost all $n$-faced near-simple quadrangulations have no nc-cycles of length two or four. When $g=0$, there is nothing to prove. For simplicity, we shall only consider non-separating nc-cycles. Separating nc-cycles can be handled more easily as in the proof of [4, Theorem 1]. Let $Q$ be a near-simple quadrangulation of genus $g>0$.

We first consider the case where $Q$ has no non-contractible 2-cycles but has a nonseparating non-contractible 4-cycle $C$. Cutting along $C$ and filling holes with disks gives a quadrangulation $Q^{\prime}$ of genus $g-1$ with two distinguished facial 4-cycles $C_{1}$ and $C_{2} . Q^{\prime}$ may have some contractible non-facial 4-cycles which correspond to the 4-cycles in $Q$ homotopic to $C$. Since $Q$ has no contractible non-facial 4-cycles, all contractible non-facial 4-cycles in $Q^{\prime}$ must contain either $C_{1}$ or $C_{2}$. 
To visualise the next step, it may be helpful to imagine that $C_{1}$ is drawn in the plane. Suppose that there are some non-facial 4-cycles containing $C_{1}$. Cutting along all these non-facial 4-cycles and filling the resulting holes with disks gives a sequence $S_{1}$ of quadrangulations. We can regard each of these as having a distinguished face as well as a root face, in order to mark the two distinct faces coming from the cuts along 4-cycles.

We must characterize the possibilities for the sequence $S_{1}$. We firstly describe this in terms of the map $M$ corresponding to the quadrangulation $Q$. The operation of cutting along $C$ in $Q$ corresponds to cutting $M$ along a closed curve through two vertices and two faces, and adding two edges $e_{1}$ and $e_{2}$ between the vertices sliced by the curve, to produce a map $M^{\prime}$. The edges $e_{1}$ and $e_{2}$ correspond to $C_{1}$ and $C_{2}$ in $Q^{\prime}$. Since $Q$ has no 2-cycles, neither does $Q^{\prime}$, and hence $M^{\prime}$ is 2-connected. Thus, by the results of Tutte [11], $M^{\prime}$ decomposes into 'cleavage units' each of which is either a 'polygon' (a cycle), a 'bond' (two vertices joined by a multiple edge), or is 3-connected. These cleavage units are joined together in a tree $T$, where two components adjacent in $T$ contain the same 'hinge' of $M^{\prime}$. Each hinge is a pair of vertices whose removal disconnects $M^{\prime}$, and two faces each incident with each of these vertices determine to a 4-cycle of $Q^{\prime}$. No two polygons are adjacent and no two bonds are adjacent. Since every contractible non-facial 4-cycle in $Q^{\prime}$ contains $C_{1}$ or $C_{2}$, no pair of vertices whose removal disconnects $M^{\prime}$ can leave $e_{1}$ and $e_{2}$ in the same planar component. It follows that in the subforest of $T$ induced by the planar cleavage units, the components containing cleavage units containing $e_{1}$ and $e_{2}$ are paths, and that all bonds and polygons have at most four edges. (This situation is similar to the decomposition of 3-connected graphs on removal of an edge, as encountered in [14]; but the situation there resembles the situation here when $e_{1}$ and $e_{2}$ share a face, in which case there can be no bonds with four edges.)

We introduce some shorthand terminology. Let $Q 3 A$ and $Q 4 A$ denote the quadrangulations which correspond to the maps which are just a 3-cycle and a 4-cycle respectively. Each of these quadrangulations has precisely one other rooting, giving the quadrangulations $Q 3 B$ and $Q 4 B$ respectively. These correspond to the maps dual to the 3-cycle and 4-cycle, which contain just a triple edge and just a quadruple edge respectively. We refer to both $Q 3 A$ and $Q 3 B$ as $Q 3$, and the same for $Q 4$.

Consideration of the fact that $Q$ is near-simple shows that the sequence of cleavage units of $M^{\prime}$ corresponding to $S_{1}$ can contain no 'polygon' or 'bond' of order greater than 4 , and that in each $Q 4$ in $S_{1}$, the distinguished face and the root face are non-adjacent.

We can now state the conditions on the sequence of quadrangulations in $S_{1}$ which follow from the properties of the decomposition of $M^{\prime}$ into cleavage units:

(1) Each of the quadrangulations in $S_{1}$ either is simple with more than four faces or is one of the four quadrangulations $Q 3 A, Q 3 B, Q 4 A$ and $Q 4 B$. 
(2) Two consecutive elements of $S_{1}$ cannot both be in $\{Q 3 A, Q 4 A\}$ nor both in $\{Q 3 B, Q 4 B\}$.

(3) Given the root face of either $Q 4 A$ or $Q 4 B$, there is only one valid choice for the distinguished face.

The 4-cycles containing $C_{2}$ give a similar sequence $S_{1}$. Therefore $Q^{\prime}$ is decomposed into two sequences of the type of $S_{1}$ and a near-simple quadrangulation of genus $g-1$ which has a distinguished face. In addition, the original rooting of $Q$ lies in one of these components. To give an upper bound for the number of maps $Q^{\prime}$, let $F_{1}(x)$ be the generating function for the sequence of consecutive $Q 3$ 's and $Q 4$ 's and let $F_{2}(x)$ be the generating function for planar simple quadrangulations with more than 4 faces. Then we have

$$
F_{1}(x)=\left(4 x^{3}+2 x^{4}\right) /\left(1-2 x-x^{2}\right),
$$

since the first element of the sequence can be of type $A$ or $B$, and from then on there is only one way to attach a $Q 4$ but two ways to attach a $Q 3$.

Since the sequence $S_{1}$ can begin with either $F_{1}$ or $F_{2}$ and end with either $F_{1}$ or $F_{2}$, we obtain the following upper bound for the counting series for a non-empty $S_{1}$ :

$$
G_{1}(x)=\left(F_{1}+x F_{2}^{\prime}+2 F_{1} F_{2}^{\prime} / x\right) /\left(1-F_{1} F_{2}^{\prime} / x^{3}\right) .
$$

Therefore the counting series for $Q^{\prime}$ is bounded by

$$
H_{1}(x)=2\left(x^{-2}\left(Q_{g-1}^{*}\right)^{\prime}\left(1+G_{1}(x)\right)^{2}\right)^{\prime} .
$$

(The factor of 2 appears because there are two ways to identify the distinguished faces $C_{1}$ and $C_{2}$ of $Q^{\prime}$ to retrieve $Q$. The outer derivative chooses the original rooting of Q.)

We now consider the case where the quadrangulation $Q$ has some non-contractible 2-cycles. By cutting along 2-cycles analogously to the 4-cycle case, but closing up the boundary of each resulting digon to form an edge, $Q$ can be decomposed into two sequences of planar quadrangulations without 2-cycles, each of which having a distinguished edge as well as a root edge, and a quad $Q^{\prime}$ of genus $g-1$ as described in case 1 (with the exception that $Q^{\prime}$ here has two distinguished edges instead of two distinguished faces). Let $S_{2}$ denote one of these planar sequences. In each element of $S_{2}$, all non-facial 4-cycles must contain either the distinguished edge or the root edge, so each element can be further decomposed into a sequence of the type of $S_{1}$ as described in the first case above. Therefore the counting series for $S_{2}$ is bounded by

$$
1 /\left(1-2 G_{1}(x)\right)
$$

where the factor of 2 is introduced in order to convert the distinguished face of one of the objects counted by $G_{1}$ into a distinguished edge. Thus the counting series for $Q^{\prime}$ 
in this case is bounded by

$$
H_{2}(x)=\left(x^{-2}\left(Q_{g-1}^{*}\right)^{\prime}\left(1 /\left(1-2 G_{1}(x)\right)\right)^{2}\right)^{\prime} .
$$

Using (4.1) and $F_{2}(x)=Q_{0}^{*}-x^{2}-2 x^{3}$ we can show (with the aid of Maple) that the denominators of (4.2) and (4.4) are not zero at $x=1 / 4$. Therefore by (3.3) the lowest terms in $R_{3}$ of (4.3) and (4.5) are $R_{3}^{4-5 g}$, which is negligible compared with $Q_{g}^{*}(x)$.

\section{3-connected maps}

The proof of Theorem 1 follows easily from Theorem 2 and the definitions of $t_{g}$ and $p_{g}$ in [1].

We now prove Corollary 1 . It was shown in [4, Theorem 1] that, if $\mathscr{F}$ is a class of maps with normal growth, then the edge-width of most maps in $\mathscr{F}_{n}(S)$ is about $\log n$. In fact, the argument used there can also be used to show that the face-width of most such maps is also about $\log n$, thus proving Corollary 1 .

Since the class of 3-connected maps on a surface has normal growth, it follows from [3, Corollary 1] that almost all of them contain any given 3-connected planar map $M$. One particular map $M$ was used in [9] to show that almost all 3-connected triangulations of the plane are not hamiltonian. The same map now suffices to complete the proof of Corollary 2.

\section{References}

[1] E. A. Bender and E. R. Canfield, 'The asymptotic number of rooted maps on a surface', J. Combin. Theory Ser. A 43 (1986), 244-257.

[2] E. A. Bender, E. R. Canfield and R. W. Robinson, 'The enumeration of maps on the torus and projective plane', Canad. Math. Bull. 31 (1988), 257-271.

[3] E. A. Bender, Z. C. Gao and L. B. Richmond, 'Submaps of maps I: general 0-1 laws', J. Combin. Theory Ser. B 55 (1992), 104-117.

[4] — , 'Almost all rooted maps have large representativities', J. Graph Theory 18 (1994), 545555.

[5] E. A. Bender and N. C. Wormald, 'The asymptotic number of rooted nonseparable maps on a surface', J. Combin. Theory Ser. A 49 (1988), 370-380.

[6] W. G. Brown, 'Enumeration of quadrangular dissections of the disk', Canad. J. Math. 17 (1965), 302-317.

[7] Z. C. Gao, 'A pattern for the asymptotic number of rooted maps on surfaces', J. Combin. Theory Ser. A 64 (1993), 246-264.

[8] R. C. Mullin and P. J. Schellenberg, 'The enumeration of c-nets via quadrangulations', J. Combin. Theory 4 (1968), 259-276. 
[9] L. B. Richmond and N. C. Wormald, 'Random triangulations of the plane', European J. Combin. 9 (1988), 61-71.

[10] N. Robertson and R. Vitray, 'Representativity of surface embeddings', Algorithms Combin. 9 (1990), 293-328.

[11] W. T. Tutte, 'A theory of 3-connected graphs', Nederl. Akad. Wetensch. Proc. Ser. B 64 (1961), $441-455$.

[12] _- 'A census of planar maps', Canad. J. Math. 15 (1963), 249-271.

[13] H. Whitney, 'Congruent graphs and the connectivity of graphs', Amer. J. Math. 54 (1932), 150-168.

[14] N. C. Wormald, 'Enumeration of labelled graphs I: 3-connected graphs', J. London Math. Soc. (2) 19 (1979), 7-12.

Center for Communications Research

4350 Executive Drive

San Diego, CA 92121

USA

Department of Combinatorics and Optimization

University of Waterloo

Waterloo, Ontario N2L3G1

Canada
Department of Combinatorics and Optimization University of Waterloo Waterloo, Ontario N2L3G1

Canada

Department of Mathematics University of Melbourne Parkville, VIC 3052 Australia

e-mail: nick@maths.mu.oz.au 